PROCEEDINGS OF THE

AMERICAN MATHEMATICAL SOCIETY

Volume 127, Number 1, January 1999, Pages 1-10

S 0002-9939(99)05006-6

\title{
ON THE EXACT LOCATION OF THE ZEROS OF CERTAIN FAMILIES OF RATIONAL PERIOD FUNCTIONS AND OTHER RELATED RATIONAL FUNCTIONS
}

\author{
ELLEN GETHNER
}

(Communicated by Dennis A. Hejhal)

\begin{abstract}
The classification of Rational Period Functions on the modular group has been of some interest recently, and was accomplished by studying the pole sets of these rational functions. We take a complex analytic point of view and begin an investigation into the location of zeros of certain families of rational period functions.
\end{abstract}

\section{INTRODUCTION}

In $[\mathrm{PR}]$, A. Parson and K. Rosen asked the following open-ended question: "What can be said about the zeros of a rational period function?" The purpose of this paper is to begin an investigation into the location of zeros of RPFs defined on $G\left(\lambda_{n}\right)$ for $n \geq 3$ and $\lambda_{n}=2 \cos \left(\frac{\pi}{n}\right)$, and to provide a generalization to another class of related rational functions. Before diving into technicalities, we begin with some motivation and history.

\section{Motivation}

In order to understand the definition of rational period function, we must first give definitions for modular form and modular integral with associated rational period function.

Definition 2.1. A function $f$ analytic in $\mathcal{H}$ is said to be an entire modular form of weight $2 k$ for $k \in \mathbf{Z}$ if

(a) $\left(\left.f\right|_{2 k} M\right)(z)=f(z)$ for all $M \in \Gamma(1)$, and

(b) $f$ has a Fourier expansion given by $\sum_{n=0}^{\infty} a_{n} e^{2 \pi i n z}$, valid for all $z \in \mathcal{H}$.

If $a_{0}=0$, then $f$ is said to be a cusp form of weight $2 k$.

Here, $\Gamma(1)$ is the Modular Group (or $P S L_{2}(\mathbf{Z})$ ), and the symbol $\left.\right|_{r}$ is the usual slash (or stroke) operator, and is defined by

$$
\left(F \mid{ }_{r} M\right)(z)=(c z+d)^{-r} F(M z),
$$

where $M=\left(\begin{array}{ll}* & * \\ c & d\end{array}\right)$ and $r \in \mathbf{R}$.

Received by the editors August 10, 1996.

1991 Mathematics Subject Classification. Primary 11F99.

The author's research at MSRI was supported in part by NSF grant DMS-9022140.

(c)1999 American Mathematical Society 
As evidence for the existence of entire modular forms of weight $2 k$, we offer the Eisenstein series $G_{2 k}(z)$, defined by

$$
G_{2 k}(z)=\sum_{\substack{m, n=-\infty \\(m, n) \neq(0,0)}}^{\infty} \frac{1}{(m+n z)^{2 k}},
$$

where, for $k>1$, the series $G_{2 k}(z)$ converges uniformly on compact subsets of $\mathcal{H}$, and therefore represents an analytic function on $\mathcal{H}$. In fact, for $k>1, G_{2 k}(z)$ is an entire modular form of weight $2 k$, and moreover, every entire modular form is a polynomial in $G_{4}(z)$ and $G_{6}(z)$. For details, see [Ap, p. 12]. When $k=1, G_{2}(z)$ does not satisfy part (a) of Definition 2.1 because $G_{2}(z)$ converges only conditionally in $\mathcal{H}$. This 'failure' will be one of the motivating forces behind the definition of Modular Integral with associated Rational Period Function.

With the above motivation in hand, we give the definition for Modular Integral with Associated Rational Period Function.

Definition 2.2. Suppose $f$ is meromorphic in $\mathcal{H}$ and satisfies

$$
\left(\left.f\right|_{2 k} S\right)(z)=f(z)
$$

and

$$
\left(\left.f\right|_{2 k} T\right)(z)=f(z)+q(z),
$$

where $k$ is an integer, $q(z)$ is a rational function, and $S=\left(\begin{array}{ll}1 & 1 \\ 0 & 1\end{array}\right)$ and $T=\left(\begin{array}{cc}0 & -1 \\ 1 & 0\end{array}\right)$ are the standard generators of $\Gamma(1)$. If, in addition, $f$ is meromorphic at $i \infty$, then $f$ is a modular integral of weight $2 k$ with associated rational period function (abbreviated as RPF) $q(z)$.

\section{History of RATiOnAL PERIOD FUnCTIONS}

In [Kn1] and [Kn4], Knopp showed that a rational function $q$ is an RPF of weight $2 k$ if and only if the following two functional equations are satisfied:

$$
\left.q\right|_{2 k} T+q=0
$$

and

$$
\left.q\right|_{2 k}(S T)^{2}+\left.q\right|_{2 k}(S T)+q=0 .
$$

Moreover, in [Kn1], Knopp added to the collection of known RPFs (those with a pole at zero, and the Eichler polynomials, which have poles at $\infty$ ) by giving the first example of RPFs with real quadratic irrational poles, namely for $k$ a positive odd integer,

$$
q(z)=\frac{1}{\left(z^{2}-z-1\right)^{k}}+\frac{1}{\left(z^{2}+z-1\right)^{k}},
$$

and proved that $q$ is never an eigenfunction of $\widehat{T}_{2 k}(n)$, the induced Hecke operator. Note that the poles of $q$ are $\frac{ \pm(1 \pm \sqrt{5})}{2}$, which are in $\mathbf{Q}(\sqrt{5})$. 
In [Kn2], using functional equations (5) and (6), Knopp completely classified RPFs with poles only at 0 as follows. If $q$ is an RPF of weight $2 k>0$ with poles only in $\mathbf{Q}$, then

$$
q(z)= \begin{cases}b_{0}\left(1-\frac{1}{z^{2 k}}\right) & \text { if } k>1 \\ b_{0}\left(1-\frac{1}{z^{2}}\right)+\frac{b_{1}}{z} & \text { if } k=1\end{cases}
$$

for some $b_{0}, b_{1} \in \mathbf{C}$.

If $k=1, b_{0}=0$, and $b_{1} \neq 0$, then $q(z)=\frac{b_{1}}{z}$ is the RPF associated with a suitable multiple of $G_{2}$, the Eisenstein series of weight 2 .

What ultimately turned out to be of greater consequence and interest in [Kn2] was that Knopp proved that the finite non-zero poles of RPFs are necessarily fixed points of hyperbolic elements of $\Gamma(1)$ and therefore are real quadratic irrational numbers.

This elegant revelation about poles provoked a variety of papers on the subject of RPFs. For our purposes, it is a selection of those papers describing RPFs with poles that are fixed points of hyperbolic elements of the appropriate group (certain Hecke groups which will be described later) that are of primary interest. For convenience, we call such a pole a hyperbolic pole.

In $[\mathrm{PR}]$, A. Parson and K. Rosen found examples of RPFs with poles in $\mathbf{Q}(\sqrt{3})$ and $\mathbf{Q}(\sqrt{21})$, and showed that these examples were never eigenfunctions of the induced Hecke operator $\widehat{T}_{2 k}(n)$.

In [Ha], J. Hawkins began a study of quadratic irrational poles of RPFs by introducing the notion of irreducible systems of poles as minimal sets of quadratic irrational numbers, that are arrived at because of the nature of the functional equations (5) and (6). For example, if $z_{0} \neq 0$ is a finite pole of an $\operatorname{RPF} q$, then by functional equation (5), $T z_{0}=\frac{-1}{z_{0}}$ must also be a pole of $q$. Hawkins also showed that for $k<0$, an RPF $q$ of weight $2 k$ must be a polynomial, and for $k=0, q$ must be identically 0 . In other words, RPFs with finite non-zero poles must be of strictly positive weight.

In [Ch], Y-J. Choie developed two methods by which to construct RPFs of positive weight with poles in $\mathbf{Q}(\sqrt{N})$ for any positive non-square integer $N$.

In [CP1] and [CP2] Choie and Parson began a more detailed classification of RPFs of positive weight and, to do so, drew extensively from the theory of indefinite binary quadratic forms. In particular, it follows from [CP1] that

$$
\sum_{\substack{a>0>c \\ b^{2}-4 a c=D>0}} \frac{1}{\left(a z^{2}+b z+c\right)^{k}}
$$

is always an RPF of weight $2 k$ when $k>0$ is an odd integer, and it is those elegant families of RPFs on which we focus our attention for the problem of locating zeros.

In $[\mathrm{Pa}]$ and $[\mathrm{CZ}] \mathrm{RPFs}$ of positive weight have been completely classified.

Finally, for purposes of this paper, it is worth highlighting a result of M. Knopp in $[\mathrm{Kn} 4]$ in which he proves the following. The Hecke groups $G\left(\lambda_{n}\right)$ are generated by $S=\left(\begin{array}{cc}1 & \lambda_{n} \\ 0 & 1\end{array}\right)$ and $T=\left(\begin{array}{cc}0 & -1 \\ 1 & 0\end{array}\right)$, where $\lambda_{n}=2 \cos \left(\frac{\pi}{n}\right)$ for $n \geq 3$. Note that when 
$n=3$, then $G\left(\lambda_{n}\right)=\Gamma(1)$, the modular group. In [Kn4] Knopp showed that a rational function $q_{\lambda_{n}}$ is an RPF on $G\left(\lambda_{n}\right)$ if and only if the following two functional equations are satisfied:

$$
\left.q_{\lambda_{n}}\right|_{2 k} T+q_{\lambda_{n}}=0
$$

and

$$
\left.\sum_{i=1}^{n-1} q_{\lambda_{n}}\right|_{2 k}\left(S_{\lambda_{n}} T\right)^{i}+q_{\lambda_{n}}=0 .
$$

For a more comprehensive history of RPFs, see [Kn3].

\section{LOCATING THE Zeros of CERTAin FAMiLiES OF RATIONAL PERIOD FUNCTIONS}

Recall the question that acted as a catalyst for this paper: "What can be said about the zeros of an RPF?" The elegant placement of the poles of RPFs, namely quadratic irrationals in the case of the modular group, and fixed points of hyperbolic elements of the more general Hecke groups $G\left(\lambda_{n}\right)$, leads complex analysts to hope for a nice characterization of the zeros of such functions. Although the characterization of poles is simple, one cannot necessarily expect a similarly simple characterization of the zeros of such a function, as evidenced by, say, the Riemann Zeta Function. In the particular case of certain families of RPFs defined on all of the Hecke groups $G\left(\lambda_{n}\right)$, we have a characterization of the zeros, and can give their exact location, as we show in an upcoming theorem. Then we extend the result to a more general class of rational functions (which will include these particular RPFs), which we will analyze from a more algebraic point of view.

We begin by offering several infinite families of RPFs defined on the infinite family of Hecke groups $G\left(\lambda_{n}\right)$. First, as shown in [PR], the following are RPFs on $G\left(\lambda_{n}\right)$ :

$$
q_{n}(z)=\frac{1}{\left(z^{2}-\lambda_{n} z-1\right)^{k}}+\frac{1}{\left(z^{2}+\lambda_{n} z-1\right)^{k}},
$$

where $k \geq 1$ is an odd integer.

Next, the following infinite family are RPFs on $G(\sqrt{3})$ :

$$
q(z)=\frac{1}{\left(z^{2}-\frac{1}{\sqrt{3}} z-1\right)^{k}}+\frac{1}{\left(z^{2}+\frac{1}{\sqrt{3}} z-1\right)^{k}},
$$

where, again, $k \geq 1$ is an odd integer. Note that when $n=3$ the family of RPFs given in equation (12) is the original family of RPFs with quadratic irrational poles found by Knopp in [Kn1] as described in equation (7) in Section 3. Moreover, the RPFs given in equation (13) are distinct from the RPFs in equation (12) for $n=6$ because the poles of $q_{6}$ are $\frac{ \pm \sqrt{3} \pm \sqrt{7}}{2}$, whereas the poles of $q$ are $\frac{ \pm \sqrt{3} \pm \sqrt{39}}{6}$.

\section{The EXACT LOCATION OF ZEROS OF CERTAin RATIONAL PERIOD FUNCTIONS}

With the examples of Section 4 in mind, we are now ready to compute the exact location of the zeros of these families of functions.

Proposition 5.1. Let $f(z)=\frac{1}{\left(z^{2}-b z-1\right)^{k}}+\frac{1}{\left(z^{2}+b z-1\right)^{k}}$ for any $b \neq 0$ in $\mathbf{R}$. If $f(x+i y)=0$, then either $x=0$ or else $x^{2}+y^{2}=1$. In other words, the zeros of $f$ lie on the unit circle or on the imaginary axis. 
Proof. Suppose $f(x+i y)=0$. Then

$$
\frac{1}{\left((x+i y)^{2}-b(x+i y)-1\right)^{k}}+\frac{1}{\left((x+i y)^{2}+b(x+i y)-1\right)^{k}}=0
$$

implies that

$$
\left(\frac{(x+i y)^{2}-b(x+i y)-1}{(x+i y)^{2}+b(x+i y)-1}\right)^{k}=-1 .
$$

Alternatively,

$$
\left|(x+i y)^{2}-b(x+i y)-1\right|^{2}=\left|(x+i y)^{2}+b(x+i y)-1\right|^{2} .
$$

We simplify equation (16) and get

$$
2 b x\left(x^{2}-y^{2}-1\right)+4 b x y^{2}=-2 b x\left(x^{2}-y^{2}-1\right)-4 b x y^{2},
$$

which gives

$$
b x\left(x^{2}+y^{2}-1\right)=0 .
$$

Therefore, $x=0$ or $x^{2}+y^{2}=1$ since $b \neq 0$.

Once we know the general location of the zeros of functions of the form of $f$ as given in Proposition 5.1, we can explicitly compute the zeros of those functions as is shown in the next corollary.

Corollary 5.2. With $k \geq 1$ odd and the same hypotheses on $f$ as in Proposition 5.1, the zeros of $f$ are given as follows.

(a) The purely imaginary zeros of $f$ are of the form $y_{s} i$, where

$$
y_{s}=\frac{-b \tan \left(\frac{\pi s}{k}\right) \pm \sqrt{b^{2} \tan ^{2}\left(\frac{\pi s}{k}\right)-4}}{2}
$$

for all integers $s$ such that $1 \leq s \leq k-1$ and $\left|\tan \left(\frac{\pi s}{k}\right)\right| \geq \frac{2}{b}$.

(b) The zeros of modulus 1 of $f$ are of the form $z_{s}= \pm \sqrt{1-y_{s}^{2}}+i y_{s}$, where

$$
y_{s}=\frac{b}{2} \tan \left(\frac{\pi s}{k}\right)
$$

for all integers $s$ such that $0 \leq s \leq k-1$ and $\left|\tan \left(\frac{\pi s}{k}\right)\right| \leq \frac{2}{b}$.

Proof. (a) By equation (15) with $k$ odd, we have

$$
\left(\frac{(i y)^{2}-b(i y)-1}{-(i y)^{2}-b(i y)+1}\right)^{k}=\left(\frac{-y^{2}-b(i y)-1}{y^{2}-b(i y)+1}\right)^{k}=1
$$

or, alternatively, that $\frac{-y^{2}-b(i y)-1}{y^{2}-b(i y)+1}$ is a $k$ th root of unity. In that case, to find $y$, we must solve

$$
\frac{-y^{2}-b(i y)-1}{y^{2}-b(i y)+1}=\cos \left(\frac{2 \pi s}{k}\right)+i \sin \left(\frac{2 \pi s}{k}\right)
$$

for integers $s$ such that $0 \leq s \leq k-1$. That is, we must determine which $k$ th roots of unity provide a real solution for $y$ in equation (20). With this in mind, we 
rewrite equation $(20)$ as

$$
\begin{aligned}
y^{2}(1 & \left.+\cos \left(\frac{2 \pi s}{k}\right)\right)+y b \sin \left(\frac{2 \pi s}{k}\right)+\left(1+\cos \left(\frac{2 \pi s}{k}\right)\right) \\
& =i\left(y^{2} \sin \left(\frac{2 \pi s}{k}\right)+y b\left(1-\cos \left(\frac{2 \pi s}{k}\right)\right)+\sin \left(\frac{2 \pi s}{k}\right)\right) .
\end{aligned}
$$

Therefore,

$$
y^{2}\left(1+\cos \left(\frac{2 \pi s}{k}\right)\right)+y b \sin \left(\frac{2 \pi s}{k}\right)+\left(1+\cos \left(\frac{2 \pi s}{k}\right)\right)=0
$$

and

$$
y^{2} \sin \left(\frac{2 \pi s}{k}\right)+y b\left(1-\cos \left(\frac{2 \pi s}{k}\right)\right)+\sin \left(\frac{2 \pi s}{k}\right)=0 .
$$

When $s=0$, equation (22) has no real solutions, and equation (23) provides no information about $y$. However, when $s \neq 0$, we can rewrite (22) as

$$
y^{2}+b\left(\frac{\sin \left(\frac{2 \pi s}{k}\right)}{1+\cos \left(\frac{2 \pi s}{k}\right)}\right) y+1=0
$$

since $1+\cos \left(\frac{2 \pi s}{k}\right) \neq 0$ for $k$ odd. Similarly, we can rewrite (23) as

$$
y^{2}+b\left(\frac{1-\cos \left(\frac{2 \pi s}{k}\right)}{\sin \left(\frac{2 \pi s}{k}\right)}\right) y+1=0
$$

$\operatorname{since} \sin \left(\frac{2 \pi s}{k}\right) \neq 0$ for $k$ odd. Note that equation (24) is the same as equation (25) because

$$
\frac{\sin \left(\frac{2 \pi s}{k}\right)}{1+\cos \left(\frac{2 \pi s}{k}\right)}=\frac{1-\cos \left(\frac{2 \pi s}{k}\right)}{\sin \left(\frac{2 \pi s}{k}\right)}=\tan \left(\frac{\pi s}{k}\right)
$$

when $s \neq 0$. Therefore, we must determine for which $s$

$$
y^{2}+b \tan \left(\frac{\pi s}{k}\right) y+1=0
$$

gives a real solution for $y$. Specifically,

$$
y=\frac{-b \tan \left(\frac{\pi s}{k}\right)+\sqrt{b^{2} \tan ^{2}\left(\frac{\pi s}{k}\right)-4}}{2}
$$

and thus $y$ is real for those $s$ satisfying $\left|\tan \left(\frac{\pi s}{k}\right)\right| \geq \frac{2}{b}$, as claimed.

(b) The proof is similar in spirit to that of (a).

Note that in (b), $x= \pm 1$ are zeros (of modulus 1) of $f$, which arise when $s=0$, and in fact correspond to 1 , the trivial $k$ th root of unity. In the case when $b=1$, it is not hard to show that $\left|\tan \left(\frac{\pi s}{k}\right)\right|=2$ cannot occur. Hence, there is no overlap in the solutions given by (a) and (b). Therefore, each $k$ th root of unity provides two zeros, and thus in total, we have found $2 k$ zeros, which, in fact, can be easily shown to be distinct. These account for all of the zeros of $f$ because solving equation (15) is equivalent to finding the roots of a polynomial of degree $2 k$. On the other hand, if for some $b$ we can have $\left|\tan \left(\frac{\pi s}{k}\right)\right|=\frac{2}{b}$, then $\pm i$ occur as solutions in both (a) and (b). Since we don't know about the multiplicity, we have listed only $2 k-2$ zeros. It seems quite likely that $\left|\tan \left(\frac{\pi s}{k}\right)\right|=\frac{2}{b}$ never occurs for the particular values of $b$ given in (12) and (13), but this still remains open. 
Note also that, except when $s=0$, the zeros of modulus 1 obtained in (b) come in groups of four, namely $\left\{ \pm \sqrt{1-y_{s}^{2}} \pm i y_{s}\right\}$. Similarly, the purely imaginary zeros can be grouped in pairs $\left\{y_{s} i, y_{k-s} i\right\}=\left\{ \pm y_{s} i\right\}$, because $\tan \left(\frac{\pi s}{k}\right)=-\tan \left(\frac{\pi(k-s)}{k}\right)$. This accounts for some of the beautiful symmetry in the graphs of the zeros of functions like $f$.

Since the families of RPFs given in (12) and (13) all satisfy the hypotheses of Proposition 5.1, Corollary 5.2 provides an explicit description of their zeros.

In fact, the results of this section also apply to the families of RPFs in (12) and (13) with $k$ a negative odd integer. Under such circumstances, these RPFs are polynomials (in the literature, these are referred to as Eichler polynomials), but in the computations in Proposition 5.1 we replace $k$ with $-k$.

\section{An algebraic point of View}

Consider again the family of RPFs

$$
q_{k}(z)=\frac{1}{\left(z^{2}-z-1\right)^{k}}+\frac{1}{\left(z^{2}+z-1\right)^{k}}
$$

and let

$$
P_{1}(X, Y)=X^{2}-X Y-Y^{2} \quad \text { and } \quad P_{2}(X, Y)=X^{2}+X Y-Y^{2} .
$$

Note that indefinite binary quadratic forms $P_{1}(X, Y)$ and $P_{2}(X, Y)$ are equivalent under the action of $\Gamma(1)$ in the narrow sense because $\left.P_{1}\right|_{2 k}=P_{2}$. Note also that $q_{k}(z)=\frac{1}{P_{1}(z, 1)^{k}}+\frac{1}{P_{2}(z, 1)^{k}}$. Thus we see that the two terms of the RPF $q_{k}(z)$ are related by a group action of $\Gamma(1)$ (for more on this, see [Pa], [CP1], [CZ], [Bu], and $[\mathrm{Za}])$. By following the reasoning and computations in Section 5 , we can prove the following theorem.

Theorem 6.1. Let $P_{1}(X, Y)=a X^{2}+b X Y+c Y^{2}$ and suppose $r_{k}(z)=P_{1}(z, 1)^{-k}+$ $\left(\left.P_{1}(z, 1)\right|_{2 k} S^{n}\right)^{-k}$, where $n \in \mathbf{Z}$. Then for any odd integer $k$, the zeros of $r_{k}(x+i y)$ must lie on the line $x=\frac{-a n-b}{2 a}$ or on the circle $\left(x+\frac{a n+b}{2 a}\right)^{2}+y^{2}=\frac{a^{2} n^{2}+2 a b n+b^{2}}{4 a^{2}}$.

Moreover, with some perseverance, using Theorem 6.1 and exactly the technique used in Section 5 one can write down the exact location of the zeros of $r_{k}(z)$.

Theorem 6.1 gives some insight into the location of zeros of rational functions including and beyond our particular families of RPFs, but this is clearly just the tip of the iceberg.

\section{A CONJECTURE}

In general, not much else is known about the zeros of RPFs, as most RPFs do not satisfy the hypotheses of Proposition 5.1 or Theorem 6.1. However, after extensive experimentation with the aid of Mathematica and with the results of this paper, it appears that the graphs of the zeros of RPFs with at least one hyperbolic pole are highly symmetric. The following open-ended conjecture is suggested.

Conjecture. Suppose $q_{k}$ is an RPF of weight $2 k$ with at least one hyperbolic pole. Then for any positive odd integer $k$, if $z$ is a zero of $q_{k}$, then $z$ lies on the graph of one of a finite number of conic sections (quite likely circles and/or lines) regardless of the value chosen for $k$. In other words, if we identify an RPF by its pole set $P$, and create a family of RPFs with poles given by the set $P$ but of any weight $2 k$, then the zeros of the family of RPFs all lie on the same finite set of conic sections. 
8. Appendix: Graphs of the zeros of SOME FAmilies of RPFs We write $q_{k, D}$ in place of $\sum_{\substack{a>0>c \\ b^{2}-4 a c=D>0}} \frac{1}{\left(a z^{2}+b z+c\right)^{k}}$.

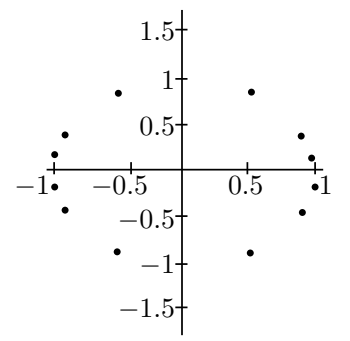

The zeros of $q_{9,5}$

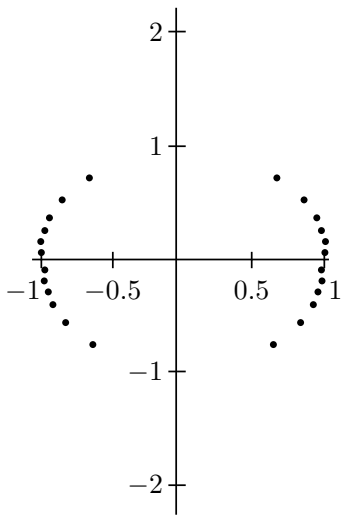

The zeros of $q_{19,5}$

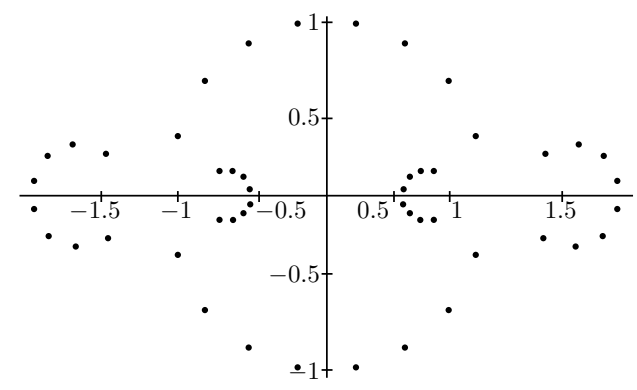

The zeros of $q_{9,5}+40\left(1-\frac{1}{z^{18}}\right)$

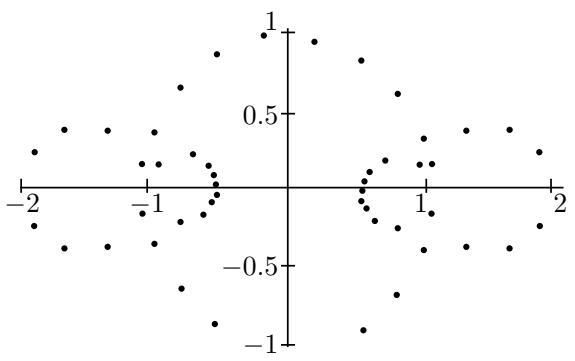

The zeros of $q_{9,5}+i\left(1-\frac{1}{z^{18}}\right)$

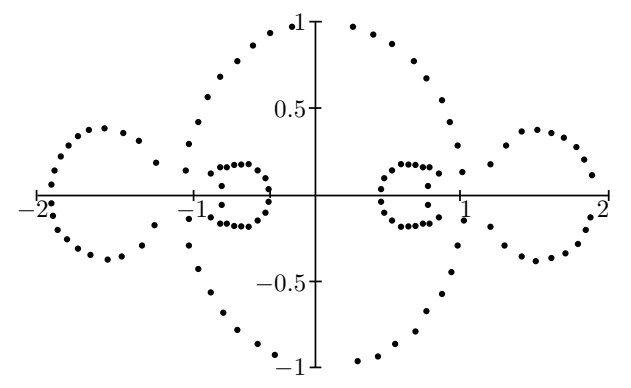

The zeros of $q_{21,5}+40\left(1-\frac{1}{z^{42}}\right)$

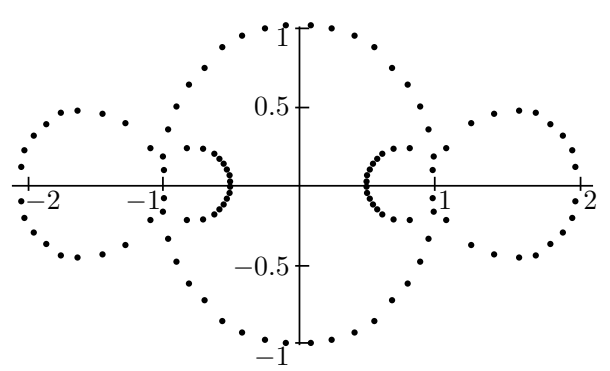

The zeros of $q_{21,5}+i\left(1-\frac{1}{z^{42}}\right)$ 


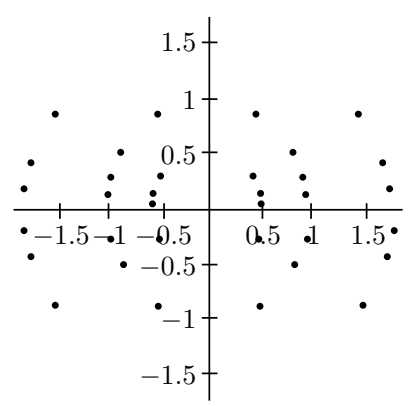

The zeros of $q_{9,8}$

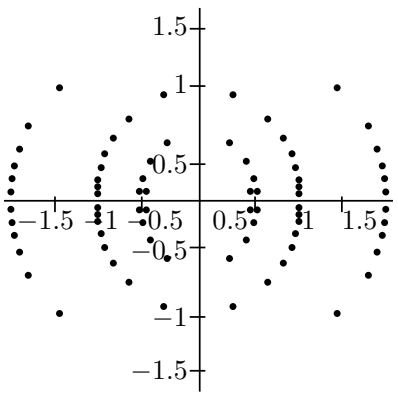

The zeros of $q_{17,8}$

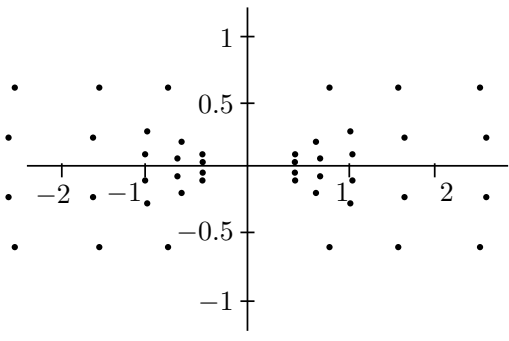

The zeros of $q_{7,13}$

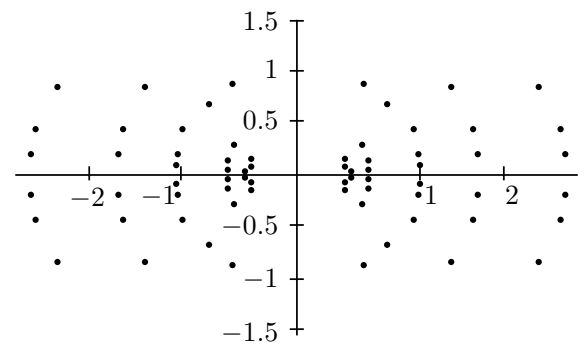

The zeros of $q_{19,13}$

\section{REFERENCES}

[Ap] T. M. Apostol, Modular Functions and Dirichlet Series in Number, 1990, Springer-Verlag, New York. MR 90j:11001

[Bu] D. A. Buell, Binary Quadratic Forms, 1989, Springer-Verlag, New York. MR 92b:11021

[Ch] Y. Choie, Rational period functions for the modular group and real quadratic fields, Ill. J. Math. 33 (1989), 495-530. MR 90i:11054

[CP1] Y. Choie and L. A. Parson, Rational period functions and indefinite binary quadratic forms, I, Math. Ann. 286 (1990), 697-707. MR 91d:11054

[CP2] Y. Choie and L. A. Parson, Rational period functions and indefinite binary quadratic forms, II, Ill, J. Math. 35 (1991), 374-400. MR 92e:11047

[CZ] Y. Choie and D. Zagier, Rational period functions for PSL $(2, \mathbf{Z})$, A Tribute to Emil Grosswald: Number Theory and Related Analysis, Contemporary Mathematics Series, Amer. Math. Soc., pp. 89-108. MR 94g:11033

[Ha] J. Hawkins, On rational period functions for the modular group, handwritten manuscript.

[Kn1] M. I. Knopp, Rational period functions of the modular group, Duke Math. J. 45 (1978), 47-62. MR 58:5522

[Kn2] M. I. Knopp, Rational period functions of the modular group, II, Glasgow Math. J. 23 (1982), 185-197. MR 83g:1001

[Kn3] M. I. Knopp, Recent developments in the theory of rational period functions, in Number Theory (New York, 1985/1988), Springer Lecture Notes in Math., 1383, 1989, 111-122. MR 91g:11051

[Kn4] M. I. Knopp, Some new results on the Eichler cohomology of automorphic forms, Bull. Amer. Math. Soc. 80 (1974), 607-632. MR 49:9193

[Pa] L. A. Parson, Rational period functions and indefinite binary quadratic forms, III, A tribute to Emil Grosswald: number theory and related analysis, 109-116, Contemp. Math., 143, Amer. Math. Soc., Providence, RI, 1993. MR 94f: 11046 
$[\mathrm{PR}]$ L. A. Parson and K. Rosen, Automorphic integrals and rational period functions for the Hecke groups, Ill. J. Math. 28 (1984), 383-396. MR 85j:11049

[Za] D. B. Zagier, Zetafunktionen und Quadratische Korper, 1981, Springer-Verlag, Heidelberg. MR 82m: 10002

Department of Mathematics, Claremont McKenna College, Claremont, California 91711

E-mail address: egethner@mckenna.edu 